\title{
Respostas à seleção de características de desempenho em tilápia-do-nilo
}

\author{
Emilia de Paiva Porto(1), Carlos Antonio Lopes de Oliveira( ${ }^{(2)}$, Elias Nunes Martins(2), Ricardo Pereira Ribeiro(2), \\ Ana Carolina Müller Conti ${ }^{(3)}$, Natali Miwa Kunita ${ }^{(4)}$, Sheila Nogueira de Oliveira(5) e Petrônio Pinheiro Porto(1)
}

\begin{abstract}
(1)Universidade Estadual do Norte do Paraná, Campus Luiz Meneghel, Rodovia BR-369, Km 54, Caixa Postal 261, Vila Maria, CEP 86360-000 Bandeirantes, PR, Brasil. E-mail: emilia@uenp.edu.br, petronio@uenp.edu (2)Universidade Estadual de Maringá, Avenida Colombo, no 5.790, CEP 87020-900 Maringá, PR, Brasil. E-mail: caloliveira@uem.br, enmartins@uem.br, rpribeiro@uem.br (3)Universidade Federal do Tocantins, Colegiado de Zootecnia, BR-153, Km 112, Caixa Postal 132, CEP 77804-970 Araguaína, TO, Brasil. E-mail: acmconti@hotmail.com (4)AquaAmérica, Rodovia BR 491, Km 158, Zona Rural, CEP 37130-000 Alfenas, MG, Brasil. E-mail: nat_kunita@hotmail.com (5)Universidade do Oeste do Paraná, Campus de Marechal Cândido Rondon, Rua Pernambuco, o 1.777, Caixa Postal 91, CEP 85960-000 Marechal Cândido Rondon, PR, Brasil. E-mail: she_uem@hotmail.com
\end{abstract}

Resumo - O objetivo deste trabalho foi estimar parâmetros genéticos e respostas direta e indireta à seleção de características de desempenho em tilápia-do-nilo (Oreochromis niloticus), bem como avaliar o impacto da seleção sobre o ganho genético. Foram utilizadas informações de tilápias-do-nilo, sob seleção em um programa de melhoramento genético, cultivadas em sistema de tanques-rede, de 2008 a 2010. Estimaram-se herdabilidades e correlações genéticas das características ganho em peso diário e, à despesca, peso, largura, altura e comprimento do tronco, além dos ganhos genéticos obtidos com as estimativas de tendências genéticas e das respostas à seleção dessas características. As estimativas de herdabilidade ficaram em torno de $30 \%$. As estimativas de correlações genéticas e de postos foram de alta magnitude. O ganho genético direto para ganho em peso diário foi de 7,9, 5,18 e 9,43\%, em 2008, 2009 e 2010, respectivamente. Os ganhos genéticos obtidos a partir das tendências genéticas foram de 6,36, 6,30,1,62, 1,65 e 1,51\%, respectivamente, para ganho em peso diário, peso, largura, altura e comprimento do tronco. $\mathrm{O}$ incremento na velocidade de crescimento por meio da seleção impacta positivamente características de desempenho em tilápia-do-nilo.

Termos para indexação: Oreochromis niloticus, correlação genética, ganho genético, seleção indireta, tendência genética.

\section{Responses to selection for performance traits in Nile tilapia}

\begin{abstract}
The objective of this work was to estimate genetic parameters and direct and indirect responses to selection for performance traits in Nile tilapia (Oreochromis niloticus), as well as to evaluate the impact of selection on genetic gain. Information about Nile tilapia, under selection in a genetic improvement program, grown in a net-tank system, from 2008 to 2010 , was used. Heritability and genetic correlations for the traits daily weight gain, and, immediately after catching, weight, width, height, and trunk length were estimated, besides the genetic gains obtained by the estimation of genetic tendencies and of the responses to selection for these traits. Heritability estimates were around 30\%. The estimates of genetic and rank correlations had high magnitude. The direct genetic gain for daily weight gain was 7.9, 5.18, and 9.43\% in 2008, 2009, and 2010, respectively. The genetic gains obtained from the genetic tendencies were $6.36,6.30,1.62,1.65$, and $1.51 \%$, respectively, for daily weight gain, and trunk weight, width, height, and length. The increased growth rate through selection positively impacts performance traits in Nile tilapia.
\end{abstract}

Index terms: Oreochromis niloticus, genetic correlation, genetic gain, indirect selection, genetic tendency.

\section{Introdução}

No melhoramento genético de tilápia-do-nilo (Oreochromis niloticus), é relevante identificar animais com rápido crescimento (Rutten et al., 2005) e com valor genético superior para características de importância econômica, a fim de alcançar ganhos genéticos em programas de seleção.
Segundo Nguyen \& Ponzoni (2006), o ganho genético normalmente é calculado pela diferença da média fenotípica entre a linha de seleção e a linha controle. No entanto, os programas de melhoramento na aquicultura são, muitas vezes, conduzidos dentro de um número muito limitado de tanques. Assim, a estimativa de tendência genética é uma alternativa de 
grande valor prático, especialmente em programas de avaliação genética em grande escala.

De acordo com Oliveira et al. (1995), o cálculo da tendência genética de uma população é uma das maneiras de se avaliar o progresso genético, o que se torna possível com a quantificação da variação anual do rebanho que tenha origem genética; para isso, é necessário que a mudança fenotípica seja decomposta nos seus componentes genéticos e de ambiente. O conhecimento da evolução genética de uma população tem importância não só para se realizarem os ajustes necessários no programa de melhoramento genético, mas, também, para que se avalie o resultado do programa de seleção que vem sendo adotado (Euclides Filho et al., 2000).

As características de crescimento são as de maior relevância econômica em programas de melhoramento de tilápias, e a taxa de crescimento é o principal objetivo de seleção nos poucos programas de melhoramento de peixe existentes (Blanck et al., 2009; Turra et al., 2010). O conhecimento da estrutura de associação entre diferentes características medidas ao longo do tempo pode fornecer informações importantes para a definição de critérios de seleção em programas de melhoramento genético de peixes (Yoshida et al., 2013b). Em programas de melhoramento genético, a seleção dos animais que serão os pais das próximas gerações depende, entre diversos fatores, das características adotadas como critérios de seleção, as quais, geralmente, são de fácil mensuração, apresentam resposta satisfatória à seleção e estão relacionadas favoravelmente aos alvos de seleção.

O objetivo deste trabalho foi estimar parâmetros genéticos e respostas direta e indireta à seleção de características de desempenho em tilápia-do-nilo, bem como avaliar o impacto da seleção.

\section{Material e Métodos}

O conjunto de dados utilizado nas análises foi cedido pelo grupo PeixeGen da Universidade Estadual de Maringá e continha informações de 6.647 animais, cultivados em tanques-rede, localizados no reservatório da Usina Hidrelétrica de Rosana, no baixo Paranapanema, na região dos municípios de Diamante do Norte, PR, e Porto Primavera, SP $\left(22^{\circ} 36^{\prime} \mathrm{S}, 52^{\circ} 50^{\prime} \mathrm{W}\right)$. Foram preditos os valores genéticos de 8.590 animais na matriz de parentesco, de cinco gerações.
As duas primeiras gerações do programa foram produzidas somente para construir as famílias, e, a partir da terceira geração, deu-se início ao processo de seleção. Para formar as famílias em cada geração, a partir de 2008, foram selecionados os melhores dois a três machos e três a quatro fêmeas de cada família. Apenas o melhor macho e as duas melhores fêmeas fizeram parte do grupo de reprodutores; os demais foram utilizados somente em caso de necessidade de substituição. Para evitar o aumento da consanguinidade e os seus efeitos adversos, impediu-se o acasalamento entre indivíduos com mais de um bisavô comum.

Foram utilizadas as informações das seguintes características: peso à despesca; largura; altura no final do período de cultivo; ganho em peso diário no período total, medido pela razão do peso final pela idade do animal ao final do período de cultivo; e comprimento do tronco, obtido pela diferença entre o comprimento padrão e o comprimento de cabeça (Tabela 1).

$\mathrm{Na}$ estimativa dos parâmetros genéticos, foram consideradas as características ganho em peso diário, peso, largura, altura e comprimento do tronco, tendo-se utilizado o modelo animal: $\mathrm{y}=\mathrm{X} \beta+\mathrm{Z}_{1} \mathrm{a}+\mathrm{Z}_{2} \mathrm{c}+\mathrm{Z}_{3} \mathrm{~W}+\varepsilon$, em que y é o vetor de observações das características analisadas, ou seja, de ganho em peso diário, peso, largura, altura e comprimento do tronco; $\mathrm{X}$ é a matriz de incidência dos efeitos ambientais identificáveis contidos no vetor $\beta$ e definidos como tanque e rede, e geração e sexo; $Z_{1}$ é a matriz de incidência dos valores genéticos contidos no vetor a; $Z_{2}$ é a matriz de incidência do efeito de ambiente comum de larvicultura (animais mantidos com as mães desde a desova até o final da estação reprodutiva) contido no vetor $\mathrm{c} ; Z_{3}$ é a matriz de incidência dos efeitos de ambiente comum de alevinagem (representantes das famílias mantidos em hapas distribuídas em locais diferentes nos tanques de alevinagem) contidos no vetor w; e $\varepsilon$ é o vetor de erros aleatórios associado ao vetor $\mathrm{y}$.

Ao se admitir que a, m, c e $\varepsilon$ apresentam distribuição conjunta normal multivariada, tem-se:

$$
\begin{gathered}
{\left[\begin{array}{l}
\mathrm{a} \\
\mathrm{m} \\
\mathrm{c} \\
\varepsilon
\end{array}\right] \sim \mathrm{NMV}\left\{\left[\begin{array}{l}
\varnothing \\
\varnothing \\
\varnothing \\
\varnothing
\end{array}\right],\left[\begin{array}{cccc}
\mathrm{A} \sigma_{\mathrm{a}}^{2} & \varnothing & \varnothing & \varnothing \\
\varnothing & \mathrm{I}_{\mathrm{k}} \sigma_{\mathrm{m}}^{2} & \varnothing & \varnothing \\
\varnothing & \varnothing & \mathrm{I}_{\mathrm{c}} \sigma_{\mathrm{c}}^{2} & \varnothing \\
\varnothing & \varnothing & \varnothing & \mathrm{I}_{\mathrm{n}} \sigma_{\varepsilon}^{2}
\end{array}\right]\right\}} \\
\mathrm{E}(\mathrm{y})=\mathrm{X} \beta ; \operatorname{Var}(\mathrm{y})=\mathrm{Z}_{1} \mathrm{AZ}_{1}{ }^{\prime} \sigma_{\mathrm{a}}^{2}+\mathrm{Z}_{2} \mathrm{Z}_{2}{ }^{\prime} \sigma_{\mathrm{m}}^{2}+\mathrm{Z}_{3} \mathrm{Z}_{3}{ }^{\prime} \sigma_{\mathrm{c}}{ }^{2}+\mathrm{I}_{\mathrm{n}} \sigma_{\varepsilon}^{2},
\end{gathered}
$$


em que A é a matriz de parentesco entre os animais; $\sigma_{\mathrm{a}}^{2}$ é a variância genética aditiva direta; $\sigma_{\mathrm{m}}^{2}, \sigma_{\mathrm{c}}^{2}$ e $\sigma_{\varepsilon}^{2}$ são as variâncias do efeito de ambiente comum de larvicultura, de ambiente comum de alevinagem e residual, respectivamente; $I_{k}$ é a matriz identidade de ordem $\mathrm{k}$, em que $\mathrm{k}$ é igual ao número de hapas de larvicultura; $\mathrm{I}_{\mathrm{c}}$ é a matriz identidade de ordem c, em que c é igual ao número de hapas de alevinagem; e $\mathrm{I}_{\mathrm{n}}$ é a matriz identidade de ordem $n$, em que $n$ é igual ao número de observações.

Foram realizadas análises uni e bicaráter a partir do sistema computacional "multiple trait Gibbs sampler for animal models" (MTGSAM, US Meat Animal Research Center, Lincoln, NE, EUA), que permite a inferência bayesiana (Van Tassel \& Van Vleck, 1995).

Nas análises, foram utilizadas cadeias de Gibbs resultantes de 500.000 ciclos, com período de descarte de 50.000 ciclos e intervalo de retirada de 30 ciclos; desse modo, foram obtidas 15.000 amostras dos componentes de variância.

Para avaliar a convergência das cadeias geradas, foi utilizado o método de Heidelberger \& Welch (1983), com uso da biblioteca Coda, implementada no sistema R (R Foundation for Statistical Computing, Viena, Áustria). A partir deste mesmo sistema, foram estimados os intervalos de credibilidade a $95 \%$ de probabilidade.

Para a predição dos valores genéticos, foram considerados os componentes de (co)variância estimados por meio do método dos modelos mistos de Henderson, implementado no sistema computacional MTGSAM.

As tendências genéticas dos valores genéticos ao longo dos 3 anos de avaliação foram estimadas em função dos anos de cultivo, por meio de equações de regressão linear, com uso da inferência bayesiana. Para isso, considerou-se que a resposta $\left(\mathrm{Y}_{\mathrm{i}}\right)$ segue

Tabela 1. Médias fenotípicas $(\mu)$ e desvios-padrões $(\sigma)$ para ganho em peso diário (GPD), peso, largura, altura e comprimento do tronco em tilápia-do-nilo (Oreochromis niloticus), nos 3 anos de avaliação.

\begin{tabular}{|c|c|c|c|c|c|c|}
\hline \multirow[t]{2}{*}{ Característica } & \multicolumn{2}{|c|}{2008} & \multicolumn{2}{|c|}{2009} & \multicolumn{2}{|c|}{2010} \\
\hline & $\mu$ & $\sigma$ & $\mu$ & $\sigma$ & $\mu$ & $\sigma$ \\
\hline GPD (g por dia) & 1,51 & 0,47 & 0,92 & 0,28 & 1,60 & 0,57 \\
\hline Peso $(g)$ & 362,50 & 110,20 & 262,67 & 83,11 & 402,42 & 140,92 \\
\hline Largura (cm) & 3,57 & 0,38 & 3,21 & 0,36 & 3,65 & 0,50 \\
\hline Altura (cm) & 7,58 & 0,83 & 7,17 & 0,86 & 8,03 & 1,07 \\
\hline Comprimento $(\mathrm{cm})$ & 14,31 & 1,27 & 12,99 & 1,35 & 14,39 & 1,71 \\
\hline
\end{tabular}

distribuição normal, isto é, $Y_{i} \sim \operatorname{normal}\left(\mu_{i}, \tau\right)$, em que $Y_{i}$ representa os valores genéticos de cada característica; e $\mu_{i}=\beta_{0}+\beta_{1} X_{i}$, em que $X_{i}$ representa as gerações, e $\beta_{0}$ e $\beta_{1}$ são consideradas distribuições a priori não informativas e independentes, com $\beta \mathrm{k} \sim$ normal $(0$, $\left.10^{-6}\right), \mathrm{k}=0,1$. Para $\tau$, também foi considerada a priori distribuição não informativa: $\tau \sim$ gama $\left(10^{-3}, 10^{-3}\right)$. A obtenção das distribuições marginais a posteriori para todos os parâmetros foi feita por meio do pacote BRugs do programa R (R Foundation for Statistical Computing, Viena, Áustria), que permite inferência bayesiana acerca dos parâmetros da equação. Foram gerados 11.000 valores pelo processo Monte Carlo-Markov chain (MCMC), tendo-se considerado o período de descarte amostral de 1.000 valores iniciais; dessa forma, a amostra final conteve 10.000 valores gerados para $\beta_{0}$ e $\beta_{1}$. A convergência das cadeias foi verificada por meio do pacote Coda do programa $R$, pelo critério de Heidelberger \& Welch (1983).

Os ganhos genéticos acumulados ao longo dos 3 anos de seleção foram calculados por meio da razão entre os coeficientes de regressão linear e as médias fenotípicas de cada característica estudada (\%), conforme proposto por Forni et al. (2007).

Os animais foram classificados de acordo com o valor genético predito para cada característica nas análises bicaráter, e, a partir da combinação das classificações para características de desempenho e de forma, foram estimadas as correlações de postos de Spearman.

Também foram estimadas, para cada ano de cultivo, as respostas direta e indireta à seleção para ganho em peso diário (GPD). Essas respostas foram calculadas com diferentes fórmulas de ganho genético $(\Delta \mathrm{g})$.

Para ganho genético direto, utilizou-se a equação,

$$
\Delta \mathrm{g}_{\mathrm{X}}=\left[\left(\overline{\mathrm{a}}_{\mathrm{m}}+\overline{\mathrm{a}}_{\mathrm{f}}\right) / 2\right]-\overline{\mathrm{a}}_{\mathrm{p}},
$$

em que: X é característica sob seleção direta; $\overline{\mathrm{a}}_{\mathrm{m}}$ é a média do valor genético dos machos selecionados; $\overline{\mathrm{a}}_{\mathrm{f}}$ é a média do valor genético das fêmeas selecionadas; e $\overline{\mathrm{a}}_{\mathrm{p}}$ é a média do valor genético da população.

Já o ganho genético indireto foi calculado como, $\Delta \mathrm{g}_{\mathrm{Y} / \mathrm{X}}=\Delta \mathrm{g}_{\mathrm{X}} \times \mathrm{b}_{1}$, em que Y: é a característica sob seleção indireta; $\Delta \mathrm{g}_{\mathrm{X}}$ é o ganho genético direto da característica sob seleção direta; e $\mathrm{b}_{1}=\sigma_{\mathrm{aX} \times \mathrm{Y}} / \sigma_{\mathrm{aX}}^{2}$, em que $\sigma_{\mathrm{aX} \times \mathrm{Y}}$ é a covariância genética aditiva entre a característica sob seleção direta e a característica sob seleção indireta, e $\sigma_{\mathrm{ax}}^{2}$ é a variância genética aditiva da característica sob seleção direta.

Pesq. agropec. bras., Brasília, v.50, n.9, p.745-752, set. 2015 DOI: $10.1590 / \mathrm{S} 0100-204 \mathrm{X} 2015000900002$ 


\section{Resultados e Discussão}

O grau de correspondência entre o valor fenotípico e o genético foi de média magnitude para as características analisadas (Tabela 2). Portanto, estas características apresentaram importância relativa das diferenças genéticas herdáveis na variação total e, consequentemente, resposta à seleção. As amplitudes dos intervalos de credibilidade das herdabilidades de todas as características foram pequenas, o que indica precisão das estimativas.

O valor da herdabilidade estimado no presente trabalho para peso à despesca foi superior ao encontrado por Santos et al. (2011), de 0,21. Estes autores utilizaram informações da primeira geração de seleção de tilápia-do-nilo da variedade GIFT, selecionadas nas condições de cultivo do Brasil, no Estado do Paraná; porém, os modelos estatísticos utilizados na estimação foram diferentes, pois os autores consideraram apenas um efeito comum, designado de efeito de ambiente comum materno.

Charo-Karisa et al. (2007) observaram estimativas de herdabilidade para peso à despesca em tilápias, cultivadas em viveiros de terra, entre 0,38 e 0,60 . Em situações de cultivo controladas, Charo-Karisa et al. (2005) testaram a resistência de tilápias às baixas temperaturas e estimaram herdabilidade de 0,10 para peso corporal. Estes resultados diferem dos obtidos no presente trabalho, o que indica influência do ambiente na expressão da característica, uma vez que os dados

Tabela 2. Valores de herdabilidade $\left(\mathrm{h}^{2}\right)$, ambiente comum de larvicultura $\left(\mathrm{C}^{2}\right)$, ambiente comum de alevinagem $\left(\mathrm{W}^{2}\right)$ e variância fenotípica $\left(\sigma^{2}\right)$, bem como respectivos intervalos de credibilidade, entre parênteses, para ganho em peso diário (GPD), peso, largura, altura e comprimento do tronco em tilápia-do-nilo (Oreochromis niloticus), nas análises unicaráter.

\begin{tabular}{lcccc}
\hline Característica & $\mathrm{h}^{2}$ & $\mathrm{C}^{2}$ & $\mathrm{~W}^{2}$ & $\sigma^{2}$ \\
\hline GPD & 0,32 & 0,14 & 0,03 & 0,19 \\
& $(0,19-0,45)$ & $(0,09-0,20)$ & $(0,02-0,04)$ & $(0,18-0,21)$ \\
Peso & 0,32 & 0,14 & 0,03 & 11.914 \\
& $(0,20-0,46)$ & $(0,09-0,19)$ & $(0,02-0,04)$ & $(10.990-13.050)$ \\
Largura & 0,29 & 0,07 & 0,02 & 0,16 \\
& $(0,18-0,41)$ & $(0,04-0,11)$ & $(0,01-0,03)$ & $(0,15-0,18)$ \\
Altura & 0,27 & 0,12 & 0,02 & 0,77 \\
& $(0,17-0,40)$ & $(0,08-0,17)$ & $(0,01-0,04)$ & $(0,71-0,82)$ \\
Comprimento & 0,29 & 0,12 & 0,02 & 1,93 \\
& $(0,19-0,41)$ & $(0,08-0,17)$ & $(0,01-0,04)$ & $(1,80-2,10)$ \\
\hline
\end{tabular}

utilizados no presente trabalho não provieram de ambientes controlados.

Em progênies de uma população baseada no pedigree da sexta geração de GIFT, geradas em 2002 e 2003, na Malásia, Ponzoni et al. (2005) encontraram herdabilidade de 0,34 para peso corporal. No entanto, esses autores também consideraram apenas o ambiente comum materno. Isso indica que, mesmo após várias gerações de seleção, a população manteve variação genética significativa, o que pode garantir a eficiência de um programa de seleção por muito mais tempo. Rutten et al. (2005), a partir de mensurações corporais de tilápia-do-nilo para seleção quanto às características de filé, estimaram valores de herdabilidade para peso corporal e largura de 0,26 e 0,25 , respectivamente, próximos aos obtidos no presente trabalho.

Ao avaliar informações da geração de seleção de tilápias-do-nilo da variedade GIFT, de 2009, pertencentes ao programa de melhoramento da Universidade Estadual de Maringá, Yoshida et al. (2013a) e Kunita et al. (2013) observaram valores de herdabilidade que variaram de 0,12 a 0,14 e de 0,08 a 0,12 , respectivamente, para peso, ganho em peso diário e altura, que foram inferiores aos do presente trabalho. Segundo Kunita et al. (2013), a utilização de informações de apenas um ciclo de produção e de uma estrutura de parentesco constituída apenas de informações entre pais e filhos e entre irmãos pode ter reduzido a capacidade de explicação da variância total pelo componente genético aditivo, o que resultou em estimativas de herdabilidade de baixa magnitude para as características avaliadas.

A participação do efeito comum de ambiente de larvicultura na variação total foi de 7 a $14 \%$ (Tabela 2), o que indica a importância desse efeito como causa de diferenças entre os animais. No presente trabalho, não houve incubação artificial, e os alevinos foram mantidos com as mães até o final da estação de acasalamento. Dessa forma, o número de alevinos produzidos, o tempo de permanência com a mãe e os cuidados parentais, além dos efeitos genéticos aditivos e não aditivos, são importantes fontes de diferenciação entre famílias de irmãos; assim, a desconsideração desses efeitos no modelo acarretaria diminuição na acurácia da avaliação genética. Para Yoshida et al. (2013a), medidas devem ser tomadas para reduzir a participação relativa do ambiente comum de larvicultura na variação total das características avaliadas, uma vez que diferenças 
ambientais no período larval resultam em diferenças no desempenho dos animais em idades próximas ao abate; os autores recomendam a incubação artificial como prática eficaz para promover essa redução.

$\mathrm{O}$ efeito comum de ambiente de alevinagem foi menor que $3 \%$ para todas as características (Tabela 2 ); contudo, de acordo com os intervalos de credibilidade, esses valores são diferentes de zero e, portanto, devem ser considerados no modelo. A manutenção de grupos de irmãos completos nas mesmas condições ambientais (hapas de alevinagem) pode ter resultado em menores diferenças entre o grupo de irmãos completos e aumentado a variabilidade entre famílias.

No presente trabalho, o efeito de ambiente comum foi dividido em dois componentes: ambiente comum de larvicultura e ambiente comum de alevinagem. Entretanto, em alguns estudos (Santos et al., 2011; Charo-Karisa et al., 2007; Ponzoni et al., 2005), temse considerado apenas o efeito de ambiente comum materno, que se caracteriza pela permanência dos alevinos da mesma família em um mesmo ambiente até atingirem o peso para serem identificados por meio de chips. A soma da participação relativa dos dois ambientes comuns (Tabela 2) se aproxima da dos valores de ambientes comuns maternos observados por Santos et al. (2011), que trabalharam com tilápias cultivadas em tanques-rede e relataram valores de 0,21 e 0,24 para peso à despesca, em análises uni e bicaráter, respectivamente. Charo-Karisa et al. (2007), em viveiros de terra, obtiveram valores da participação do efeito comum materno entre 0,08 e 0,11 para peso à despesca, enquanto Ponzoni et al. (2005), ao avaliar a sexta geração de GIFT na Malásia, estimaram em 0,15 a participação desse efeito na variação total do peso corporal.

Já Yoshida et al. (2013a) separaram os efeitos de ambiente comum de larvicultura e de alevinagem. Para o efeito comum de ambiente de larvicultura, os autores encontraram valores de $6,4,8,4,2$ e $3,8 \%$ para peso, ganho em peso diário, largura e altura, respectivamente, e para o efeito comum de ambiente de alevinagem, valores de 2,5, 1,9, 2,6 e 1,9\% para peso, ganho em peso diário, largura e altura, respectivamente.

As tendências genéticas dos efeitos diretos das características ganho em peso diário, peso, largura, altura e comprimento do tronco foram de: $0,089 \mathrm{~g}$ por dia, 22,24 g, $0,057 \mathrm{~cm}, 0,13 \mathrm{~cm}$ e $0,21 \mathrm{~cm}$, respectivamente (Figura 1). Em termos de mudança genética anual, esses resultados representaram incrementos de 6,36, 6,3, 1,62, 1,65 e 1,51\%, respectivamente, nas médias de ganho em peso diário, peso, largura, altura e comprimento do tronco, o que mostra que estas características podem ser correlacionadas geneticamente (Tabela 3 ).

Em estudo com tilápias GIFT, na China, Thodesen et al. (2011) avaliaram seis gerações de seleção com base em índice de seleção, valores genéticos individuais e de família relativos ao crescimento e ao rendimento de filé, respectivamente, e estimaram, por meio da tendência genética, frequentista, ganhos genéticos anuais de $11,4 \%$ para peso corporal.

Houve aumento nos valores genéticos preditos ao longo das três gerações de seleção para ganho em peso diário, peso, largura, altura e comprimento do tronco, associados às estimativas das importâncias relativas das
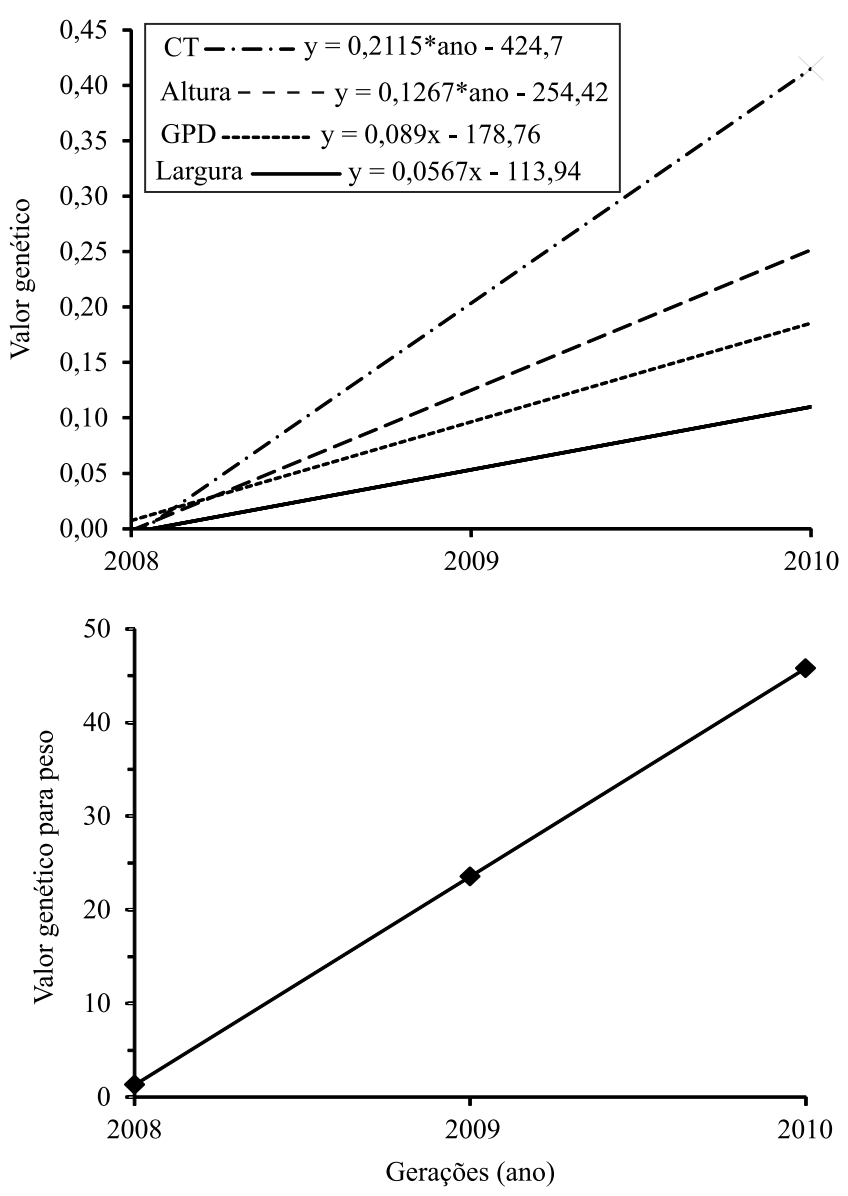

Figura 1. Tendência genética de ganho em peso diário (GPD), peso, largura, altura e comprimento do tronco (CT) em tilápia-do-nilo (Oreochromis niloticus). 
diferenças genéticas herdáveis na variação observada nessas características. Isso é indicativo da manutenção das respostas à seleção em programa de seleção por muito mais tempo, desde que o tamanho efetivo da população seja mantido elevado e os acasalamentos consanguíneos sejam controlados.

O uso de ganho em peso diário como critério de seleção nas estimativas de correlações genéticas pode impactar o peso à despesca e a largura do corpo dos animais de maneira mais expressiva do que a altura e o comprimento do tronco. As correlações genéticas estimadas entre peso e largura, e entre altura e comprimento do tronco são indicativas de que a seleção para peso pode resultar em aumento na altura e no comprimento do tronco (Tabela 4).

As altas correlações genéticas obtidas no presente trabalho são indicativas de que grande parte dos genes de ação aditiva que influencia a característica ganho em peso diário também influencia positivamente as demais características avaliadas. A mesma resposta ocorre entre as outras associações analisadas; desse modo, a seleção para qualquer uma dessas características deve resultar em progresso genético nas demais.

As estimativas de correlações genéticas e fenotípicas obtidas no presente trabalho foram semelhantes às reportadas por Nguyen et al. (2007), Charo-Karisa et al. (2007) e Rutten et al. (2005), de 0,84 a 0,99 para correlações genéticas, e de 0,76 a 0,90 para as fenotípicas. Para as associações ganho em peso diário e peso, ganho em peso diário e altura, e peso e altura, Kunita et al. (2013) estimaram correlações genéticas de $0,94,0,76$ e 0,70 , respectivamente, e correlações fenotípicas de 0,96, 0,85 e 0,86, respectivamente.

Pequenas alterações nas classificações dos animais, ao se utilizar a característica ganho em peso diário como critério de seleção, foram observadas com os valores das correlações de Spearman. As comparações das classificações de ganho em peso diário com as demais características apontaram correlação superior a $90 \%$, com exceção de comprimento do tronco, com correlação de 77\% (Tabela 5).

As correlações de Spearman estimadas no presente trabalho foram próximas às obtidas por Kunita et al. (2013), para ganho em peso diário e peso $(0,99)$, e para ganho em peso diário e altura $(0,94)$. Coeficientes de correlações de Spearman de ordem inferiores a 0,70 podem resultar em alterações no ranking dos animais, o que compromete a seleção (Crews Jr. \& Franke, 1998).

As estimativas de correlação de Spearman confirmaram os resultados da correlação genética. Isso indica que a seleção para velocidade de crescimento com uso do ganho em peso diário como critério de seleção pode promover a seleção dos animais que apresentam genótipos superiores quanto ao peso e às características de forma.

O ganho genético da geração de 2010 superou o da geração de 2008, ao se considerar que, em 2009, o ganho genético foi inferior para todas as características (Tabela 6). Isso pode ser explicado por problemas ocorridos na alimentação em 2009, o que pode ter influenciado a expressão genética das características avaliadas e prejudicado o ganho genético.

Em pesquisa com duas gerações de tilápia-do-nilo ao longo de três épocas de desova (2002, 2003 e 2004), Rezk et al. (2009) estimaram o ganho genético para peso à despesca em 6,64 e 6,69\%, a partir das diferenças dos valores genéticos entre as gerações de 2002 e 2003, e de 2003 e 2004, respectivamente. Já Ponzoni et al. (2005), ao avaliar progênies geradas de uma população da sexta geração de GIFT na Malásia, em 2002 e 2003, estimaram o ganho genético do peso vivo em $8,7 \%$ aos 7 meses de idade aproximadamente, tendo comparado os valores genéticos entre os descendentes de 2002 e os selecionados em 2003.

Tabela 3. Coeficientes de regressão $\left(\beta_{1}\right)$ e respectivos intervalos de credibilidade (IC), médias fenotípicas ( $\mu$ ) e ganhos genéticos $(\Delta \mathrm{g})$ para as características ganho em peso diário (GPD), peso, largura, altura e comprimento do tronco em tilápia-do-nilo (Oreochromis niloticus), da variedade GIFT, sob seleção quanto ao GPD.

\begin{tabular}{|c|c|c|c|c|c|}
\hline \multirow[b]{2}{*}{ Parâmetro } & \multicolumn{5}{|c|}{ Característica } \\
\hline & GPD & Peso & Largura & Altura & Comprimento \\
\hline$\overline{\beta_{1}}$ & 0,089 & 22,24 & 0,05674 & 0,12670 & 0,2115 \\
\hline $\mathrm{IC}^{(1)}$ & $0,088-0,09$ & $22,08-22,42$ & $0,0567-0,05678$ & $0,12662-0,12678$ & $0,2113-0,2116$ \\
\hline$\mu$ & 1,40 & 353,11 & 3,51 & 7,66 & 13,99 \\
\hline$\Delta \mathrm{g}$ & $6,36 \%$ & $6,30 \%$ & $1,62 \%$ & $1,65 \%$ & $1,51 \%$ \\
\hline
\end{tabular}

${ }^{(1)}$ Intervalo de credibilidade de 2,5 a $97,5 \%$. 
Em estudos com informações da primeira geração de seleção de tilápias-do-nilo da variedade GIFT selecionadas nas condições do Estado do Paraná, Santos et al. (2011) estimaram o ganho genético do peso à despesca, em machos e fêmeas, em 15 e $13,8 \%$, respectivamente, tendo selecionado os melhores indivíduos dentro das melhores famílias. Quando os melhores indivíduos de cada família foram selecionados, os ganhos genéticos estimados foram de 7,4 e 3,9\% em machos e fêmeas, respectivamente, resultados mais próximos aos encontrados no presente trabalho, em que também se trabalhou com a seleção dos melhores indivíduos de cada família.

Tabela 4. Herdabilidades nas análises bicaráter (na diagonal), e correlações genética (abaixo da diagonal) e fenotípica (acima da diagonal) entre as características ganho em peso diário (GPD), peso, largura, altura e comprimento do tronco em tilápia-do-nilo (Oreochromis niloticus).

\begin{tabular}{lccccc}
\hline Característica & GPD & Peso & Largura & Altura & Comprimento \\
\hline GPD & 0,26 & 0,85 & 0,77 & 0,77 & 0,72 \\
Peso & 0,99 & 0,34 & 0,60 & 0,82 & 0,83 \\
Largura & 0,91 & 0,67 & 0,28 & 0,66 & 0,70 \\
Altura & 0,81 & 0,94 & 0,68 & 0,27 & 0,76 \\
Comprimento & 0,74 & 0,91 & 0,71 & 0,81 & 0,26 \\
\hline
\end{tabular}

Tabela 5. Correlações de postos de Spearman entre as características ganho em peso diário (GPD), peso, largura, altura e comprimento do tronco em tilápia-do-nilo (Oreochromis niloticus).

\begin{tabular}{lcccc}
\hline Características & GPD & Peso & Largura & Altura \\
\hline Peso & 0,99 & & & \\
Largura & 0,99 & 0,85 & & \\
Altura & 0,91 & 0,98 & 0,83 & \\
Comprimento & 0,77 & 0,96 & 0,76 & 0,90 \\
\hline
\end{tabular}

Tabela 6. Ganho genético $(\Delta \mathrm{g})$ direto em ganho em peso diário (GPD), e ganho genético $(\Delta \mathrm{g})$ indireto em peso, largura, altura e comprimento do tronco em tilápia-do-nilo (Oreochromis niloticus), nos 3 anos de avaliação.

\begin{tabular}{lcccccc}
\hline & \multicolumn{2}{c}{$\Delta \mathrm{g}$ direto $(\%)$} & \multicolumn{4}{c}{$\Delta \mathrm{g}$ indireto (\%) } \\
\cline { 2 - 6 } \cline { 5 - 7 } Geraçân & GPD & & Peso & Largura & Altura & Comprimento \\
\hline 2008 & 7,91 & & 7,98 & 2,49 & 2,92 & 2,03 \\
2009 & 5,18 & & 4,40 & 1,11 & 1,23 & 0,89 \\
2010 & 9,43 & & 9,09 & 3,08 & 3,48 & 2,56 \\
\hline
\end{tabular}

Kunita et al. (2013) e Yoshida et al. (2013a), ao avaliar a geração de 2009 do mesmo programa de seleção, encontraram valores de ganhos genéticos direto e indireto em torno de $2 \%$ para ganho em peso diário, peso, largura e altura. Essas discrepâncias de resultados podem estar relacionadas à estrutura de dados divergentes, ao número de observações e à estrutura de parentesco utilizada na estimação dos parâmetros genéticos.

Com base nos resultados obtidos nas estimativas de correlações e respostas à seleção, foi possível observar que o processo de seleção que utiliza o ganho em peso diário como critério pode resultar em ganhos genéticos indiretos, que modificam a forma do peixe, aumentam o peso, a altura, a largura e o comprimento do tronco. Isso é de grande importância econômica para os piscicultores, uma vez que acarreta aumento direto na produção de animais com melhores carcaças e na produção em menor intervalo de tempo. No presente trabalho, o tempo médio para o animal ser abatido foi de 180 dias de cultivo, com ganho genético em relação ao ganho em peso diário de 9,43\%, em 2010, o que acarretou diminuição de aproximadamente 17 dias na idade ao abate. Assim, se a conversão alimentar não for correlacionada negativamente à característica em questão, o produtor terá maior lucro na atividade.

\section{Conclusões}

1. Há manutenção dos ganhos genéticos em velocidade de crescimento e elevadas respostas à seleção quanto a peso, largura, altura e comprimento do tronco em tilápia-do-nilo (Oreochromis niloticus).

2. A seleção para incremento na velocidade de crescimento acarreta aumento na qualidade genética e impacta positivamente outras características de interesse econômico, relacionadas à forma do corpo dos peixes.

3. Há forte correlação genética entre ganho em peso diário e peso, largura, altura e comprimento do tronco.

4. A seleção para ganho em peso diário pode conduzir a ganhos genéticos indiretos para as características peso e forma do corpo, em tilápia-do-nilo.

\section{Agradecimentos}

AogrupodepesquisaPeixeGen, peladisponibilização de parte do seu banco de dados para análise. 


\section{Referências}

BLANCK, D.V.; GASPARINO, E.; RIBEIRO, R.P.; MARQUES, D.S. Polimorfismo no gene GH1-PstI associado a características corporais de linhagens de tilápia-do-nilo. Pesquisa Agropecuária Brasileira, v.44, p.599-604, 2009. DOI: 10.1590/ S0100-204X2009000600008.

CHARO-KARISA, H.; BOVENHUIS, H.; REZK, M.A.; PONZONI, R.W.; VAN ARENDONK, J.A.M.; KOMEN, H. Phenotypic and genetic parameters for body measurements, reproductive traits and gut length of Nile tilapia (Oreochromis niloticus) selected for growth in low-input earthen ponds. Aquaculture, v.273, p.15-23, 2007. DOI: 10.1016/j. aquaculture.2007.09.011.

CHARO-KARISA, H.; REZK, M.A.; BOVENHUIS, H.; KOMEN, H. Heritability of cold tolerance in Nile tilapia, Oreochromis niloticus, juveniles. Aquaculture, v.249, p.115-123, 2005. DOI: 10.1016/j.aquaculture.2005.04.029.

CREWS JR., D.H.; FRANKE, D.E. Heterogeneity of variances for carcass traits by percentage Brahman inheritance. Journal of Animal Science, v.76, p.1803-1809, 1998.

EUCLIDES FILHO, K.; SILVA, L.O.C. da; ALVES, R.G. de O.; FIGUEIREDO, G.R. de. Tendência genética na raça Gir. Pesquisa Agropecuária Brasileira, v.35, p.787-791, 2000. DOI: 10.1590/ S0100-204X2000000400016.

FORNI, S.; FEDERICI, J.F.; ALBUQUERQUE, L.G. de. Tendências genéticas para escores visuais de conformação, precocidade e musculatura à desmama de bovinos Nelore. Revista Brasileira de Zootecnia, v.36, p.572-577, 2007. DOI: 10.1590/ S1516-35982007000300008.

HEIDELBERGER, P.; WELCH, P.D. Simulation run length control in the presence of an initial transient. Operation Research, v.31, p.1109-1144, 1983. DOI: 10.1287/opre.31.6.1109.

KUNITA, N.M.; OLIVEIRA, C.A.L.; OLIVEIRA, S.N.; YOSHIDA, G.M.; RIZZATO, G.S.; RESENDE, E.K.; RIBEIRO, R.P. Avaliação genética de características morfométricas em tilápias do Nilo cultivadas. Archivos de Zootecnia, v.62, p.555-566, 2013. DOI: $10.4321 / \mathrm{S} 0004-05922013000400008$.

NGUYEN, N.H.; KHAW, H.L.; PONZONI, R.W.; HAMZAH, A.; KAMARUZZAMAN, N. Can sexual dimorphism and body shape be altered in Nile tilapia (Oreochromis niloticus) by genetic means? Aquaculture, v.272, p.S38-S46, 2007. DOI: 10.1016/j. aquaculture.2007.08.013.

NGUYEN, N.H.; PONZONI, R.W. Perspectives from agriculture: advances in livestock breeding - implications for aquaculture genetics. NAGA, WorldFish Center Quarterly, v.29, p.39-45, 2006.

OLIVEIRA, J.A. de; LÔBO, R.B.; OLIVEIRA, H.N. de. Tendência genética em pesos e ganhos em peso de bovinos da raça
Guzerá. Pesquisa Agropecuária Brasileira, v.30, p.1355-1360, 1995.

PONZONI, R.W.; AZHAR, H.; SAADIAH, T.; NORHIDAYAT, K. Genetic parameters and response to selection for live weight in the GIFT strain of Nile tilapia (Oreochromis niloticus). Aquaculture, v.247, p.203-210, 2005. DOI: 10.1016/j.aquaculture.2005.02.020.

REZK, M.A.; PONZONI, R.W.; KHAW, H.L.; KAMEL, E.; DAWOOD, T.; JOHN, G. Selective breeding for increased body weight in a synthetic breed of Egyptian Nile tilapia, Oreochromis niloticus: response to selection and genetic parameters. Aquaculture, v.293, p.187-194, 2009. DOI: 10.1016/j. aquaculture.2009.03.019.

RUTTEN, M.J.M.; BOVENHUIS, H.; KOMEN, H. Genetic parameters for fillet traits and body measurements in Nile tilapia (Oreochromis niloticus L.). Aquaculture, v.246, p.125-132, 2005. DOI: 10.1016/j.aquaculture.2005.01.006.

SANTOS, A.I.; RIBEIRO, R.P.; VARGAS, L.; MORA, F.; ALEXANDRE FILHO, L.; FORNARI, D.C.; OLIVEIRA, S.N. de. Bayesian genetic parameters for body weight and survival of Nile tilapia farmed in Brazil. Pesquisa Agropecuária Brasileira, v.46, p.33-43, 2011. DOI: 10.1590/S0100-204X2011000100005.

THODESEN, J.; RYE, M.; WANG，Y.-X.; YANG， K.-S.; BENTSEN, H.B.; GJEDREM, T. Genetic improvement of tilapias in China: genetic parameters and selection responses in growth of Nile tilapia (Oreochromis niloticus) after six generations of multi-trait selection for growth and fillet yield. Aquaculture, v.322-323, p.51-64, 2011. DOI: 10.1016/j.aquaculture.2011.10.010.

TURRA, E.M.; OLIVEIRA, D.A.A.; TEIXEIRA, E.A.; PRADO, S.A.; MELO, D.C.; SOUSA, A.B. Uso de medidas morfométricas no melhoramento genético do rendimento de filé da tilápia do Nilo (Oreochromis niloticus). Revista Brasileia de Reprodução Animal, v.34, p.29-36, 2010.

VAN TASSEL, C.P.; VAN VLECK, L.D. Manual for use of MTGSAM: a set of FORTRAN programs to apply Gibbs sampling to animal models for variance component estimation. Lincoln: USDA, Agricultural Research Service, 1995.

YOSHIDA, G.M.; OLIVEIRA, C.A.L.; KUNITA, N.M.; OLIVEIRA, S.N.; ALEXANDRE FILHO, L.; RESENDE, E.K.; LOPERA-BARRERO, N.M.; RIBEIRO, R.P. Resposta à seleção de características de desempenho e morfométricas de tilápia-do-nilo ao longo do período de cultivo. Arquivo Brasileiro de Medicina Veterinária e Zootecnia, v.65, p.1815-1822, 2013a. DOI: $10.1590 / \mathrm{S} 0102-09352013000600032$.

YOSHIDA, G.M.; OLIVEIRA, C.A.L. de; OLIVEIRA, S.N. de; KUNITA, N.M.; RESENDE, E.K. de; ALEXANDRE FILHO, L.; RIBEIRO, R.P. Associação entre características de desempenho de tilápia-do-nilo ao longo do período de cultivo. Pesquisa Agropecuária Brasileira, v.48, p.816-824, 2013b. DOI: 10.1590/ S0100-204X2013000800002.

Recebido em 18 de março de 2015 e aprovado em 17 de setembro de 2015 